\title{
CARACTERIZACIÓN DE LA FLORA MICROBIANA ANAEROBIA DEL CIEGO EN LA POBLACIÓN AVÍCOLA ADULTA Y SANA DEL MUNICIPIO DE LOS PATIOS CON MIRAS A LA PRODUCCIÓN DE PROBIÓTICOS CON CEPAS AUTÓCTONAS DE LA REGIÓN
}

Por:

Adiel Alberto Vasquez Quijano', Jhollman Chacon Rangel2, Margarita Caballero Chaparro ${ }^{3}$

\section{RESUMEN}

Los probióticos son aquellos microorganismos vivos que, al ser agregados como suplemento en la dieta, afectan en forma beneficiosa el desarrollo de la flora microbiana no patógena en el intestino y estimulan las funciones protectoras del sistema digestivo.

En este trabajo se presentan resultados con respecto a la caracterización de la flora intestinal anaerobia predominante en aves adultas sanas del municipio de Los Patios. Se identificaron, con género y especie, 24 bacterias, 9 de las cuales no se han reportado en la literatura internacional. Se preparó un probiótico con estos microorganismos y se evaluó frente a descargas de Salmonella resultando un Factor de Protección (FP) $>$ 17.83. Se prepararon probióticos con las 24 bacterias identificadas y la flora aerobia obtenida de cada una de las granjas en estudio, se evaluó el FP y se obtuvieron resultados que mostraron un aumento promedio del FP de $54.95 \%$. Los resultados indican que la eficiencia de un probiótico está dada por el tipo de flora aerobia y anaerobia que pueda contener.

Palabras claves: Probiótico, anaerobios, aerobios, Factor de Protección, aves adultas sanas.

\section{INTRODUCCION}

El conocimiento de los efectos benéficos de algunas de las bacterias de la flora intestinal se inicia a principios de siglo con los trabajos de Metchnikoff. Desde entonces, y a lo largo de estos casi 100 años de estudio, autores muy diversos se han esforzado en conocer las distintas funciones de los microorganismos que pueblan el tracto digestivo. A pesar de ello, algunas de sus acciones no están bien precisadas. Por otra parte, una vez comprobado que algunas bacterias intestinales, adicionadas al pienso o al agua de bebida, determinaban una respuesta favorable en producción animal, se intentó enmarcarlas en un grupo específico. Sin embargo, la propia heterogeneidad de los microorganismos experimentados no facilitó este propósito. De igual forma, no se ha resuelto una denominación técnica específica que permitiera su diferenciación de otros aditivos o sustancias no biológicas, considerados con efectos estimulantes de la producción animal.

La palabra probiótico fue utilizada por $1^{a}$ vez por Lilley y Stillwell en 1965 para referirse a "una sustancia secretada por un microorganismo que estimulaba el crecimiento de otro". En 1971, Sperti definió probiótico como "extractos de tejido que estimulaban el crecimiento microbiano", Parker, en 1974, como "organismos y substancias que contribuyen para el balance microbiano intestinal". Más tarde, en 1989, Fuller modificó el concepto como "suplemento alimenticio microbiano vivo que afecta benéficamente el hospedero animal por que mejora su balance microbiano intestinal".

En producción animal intensiva, para obtener un buen rendimiento, es indispensable el uso de antibióticos, lo cual proporciona al animal una protección frente a ciertas enfermedades, al mismo tiempo, actúan como precursores de crecimiento al eliminar microorganismos no deseados que se encuentran en el tubo digestivo. Sin embargo, el problema central de usar antibióticos en dietas para animales, radica en que queda un residuo en los productos animales que al ser consumido por el hombre, produce una resistencia de microorganismos patógenos a la acción de antibióticos y por consiguiente el hombre se encuentra en condiciones más precarias de defensa ante cualquier enfermedad, especialmente bacteriana.

\footnotetext{
'Lic. Biología y Química U.F.P.S., Bacteriólogo Pontificia U. Javeriana, Especialista en computación para la educación U.AU., OTC U.F.P.S., Facultad de Ciencias Agrarias.

${ }^{2}$ Lic. Biología y Química U.F.P.S.

${ }^{3}$ Tecnóloga Agropecuaria
} 
Caracterización de la flora microbiana anaerobia del ciego en la población avícola adulta y sana del Municipio de Los Patios con miras a la producción de probióticos con cepas autóctonas de la región

Adiel Alberto Vasquez Quijano, Jhollman Chacón Rangel, Margarita Caballero Chaparro

Si con la administración de probióticos conseguimos mantener una flora intestinal en equilibrio evitaremos problemas tan frecuentes en las aves de jaula como son las diarreas y otros problemas producidos por bacterias como la Salmonella.

Pero ¿̇cual es el probiótico ideal? Las características que debe reunir son las siguientes:

- Que no resulte dañino ni productor de sustancias tóxicas.

- Los microorganismos que los componen deben adherirse fácilmente a la pared intestinal y crecer rápidamente.

- Contener bacterias que como los lactobacilos puedan transformar la lactosa en ácido láctico, consiguiéndose así tal acidez en el tubo digestivo evitando que ciertas bacterias dañinas puedan sobrevivir.

- Incluir microorganismos que elaboren vitaminas, beneficiosas y necesarias para el ave y enzimas que ayuden a la digestión.

- Que por su simple presencia física evite que microorganismos patógenos colonicen el intestino.

En este trabajo se presentan resultados con respecto a la flora intestinal anaerobia predominante en aves adultas sanas del municipio de Los Patios, donde se calcula una población aproximada de 600.000 animales. A través de información obtenida del ICA se localizaron las granjas dedicadas a la avicultura en el área de estudio. Se eligieron 16 granjas con una población avícola de aproximadamente 536.600 aves, de las cuales 425.200 (79.24\%) son ponedoras y $111.400(20.76 \%)$ pollos de engorde.

Se revisaron todos los datos obtenidos en cada una de las granjas, con el fin de seleccionar las que reunían las condiciones para el estudio, teniendo en cuenta:

- Granjas sin historial de patologías recientes.
- Granjas cuyas aves cumplían con las características óptimas de crecimiento esperadas para la edad.

De las granjas escogidas para el estudio solamente se trabajó con 9 de ellas (89.44\% de la población avícola de Los Patios) ya que las otras siete presentaban, al momento del estudio, problemas epidemiológicos con New Castle y Gumboro, quedando una población de 452.200 animales que representan un $75.37 \%$ del total de la población.

Una vez realizado el censo de la población avícola, se eligieron los animales que cumplían las condiciones para el estudio teniendo en cuenta el criterio de ser aves adultas y sanas; en total cuatro aves en la avícola con mayor población y dos aves en cada una de las otras granjas para un total de 10 aves, con las que se procedió a realizar el estudio.

Se sacrificaron cada vez dos aves por granja y se tomó el contenido de los ciegos en un caldo de tioglicolato para el análisis de la flora microbiana anaerobia.

Diagnóstico presuntivo de anaerobios: Siempre se hace indispensable un diagnóstico rápido. El aislamiento y la identificación de los anaerobios toman algunos días y aún semanas, por lo que es necesario hacer un análisis presuntivo en la muestra, basado en dos parámetros esenciales:

\section{Examen macroscópico de la muestra.}

a) Color con tendencia al pardo o negro más que de color crema, que generalmente es de origen anaerobio.

b) Olor característico.

c) Algunos microorganismos anaerobios presentan fluorescencia, como el Bacillus melaningogénicus (fluorescencia rojo brillante).

\section{Examen microscópico de la muestra.}

a) Frente a la coloración de Gram se observan 
Caracterización de la flora microbiana anaerobia del ciego en la población avícola adulta y sana del Municipio de Los Patios con miras a la producción de probióticos con cepas autóctonas de la región

Adiel Alberto Vasquez Quijano, Jhollman Chacón Rangel, Margarita Caballero Chaparro

mezclas de cocos y bacilos, gram positivos y gram negativos.

b) Algunas veces los bacilos Gram negativos presentan características fusiformes o una morfología muy pleomorfica.

c) En ocasiones los bacilos Gram negativos pueden ser enmascarados por los cocos Gram positivos y esto presenta dificultades en la interpretación de los cultivos.

d) La morfología del Actinomyces característica, semejando más cadenas de cocos que elementos bacilares.

e) Clostridium perfringens es generalmente más obvio en su morfología, bacilos Gram positivos muy gruesos con bordes recortados que recuerdan ladrillos.

\section{MATERIALES Y METODOS:}

En el proceso se utilizaron las técnicas tradicionales de laboratorio para el cultivo de anaerobios, incluyendo:

Sistema anaeróbico GasPak: Jarra anaerobia elaborada en polycarbonato de 3.5 litros de capacidad, con un porta placas (cajas de Petri) metálico.

Sobres generadores de anaerobiosis para el sistema GasPak: Sobre de metal laminado que contiene papel de filtro, una tableta de borohidruro de sodio, una de bicarbonato de sodio y ácido cítrico. Con la adición de $10 \mathrm{ml}$ de agua destilada o desionizada, el sistema produce desprendimiento de $\mathrm{H}_{2}+\mathrm{CO}_{2}$. La reacción resultante ácida evita la reabsorción del anhídrido carbónico.

Sobres indicadores de anaerobiosis: Tira de algodón impregnada en rezarzurina en sobre de metal laminado.

\section{MEDIOS DE CULTIVO PARA ANAEROBIOS:}

Caldo Tioglicolato (enriquecido): Para el crecimiento y cultivo de microorganismos anaerobios. Este medio puede utilizarse así mismo para diversos fines en el aislamiento e identificación de estos gérmenes.

Forma de actuación: Las sustancias reductoras, tioglicolato y sulfito sódico producen una cisteina anaerobiosis suficiente para los anaerobios exigentes. La elevada viscocidad del medio impide la penetración rápida de oxígeno.

\section{Medio Fluido Tioglicolato según Brewer}

Agar sangre para anaerobios según CEC: Para cultivo de microorganismos anaerobios, incluso muy exigentes y de crecimiento lento, a partir de material clínico de investigación.

Forma de actuación: Este medio de cultivo no selectivo, rico en nutrientes, garantiza tanto el cultivo de microorganismos anaerobios exigentes como de aerobios y microaerofílicos. Favorece así mismo la formación del pigmento típico en el caso de Bacteroides melaningogénicus y la formación de doble hemólisis en Clostridium perfringens.

Sistema de identificación CRYSTAL: Un micro método cualitativo que utiliza sustratos cromogénicos y convencionales modificados. El principio del sistema CRYSTAL se basa en que los paneles del mismo contienen 30 sustratos bioquímicos y enzimáticos deshidratados que se hidratan con la suspensión de bacterias en fluido del inóculo. Las pruebas se basan en la utilización y degradación microbiana de sustratos específicos detectados por varios sistemas indicadores.

Sistema de identificación IDS Rapid ANA II System: Es también un micro método cualitativo de identificación que emplea sustratos convencionales y cromogénicos para la identificación de bacterias anaerobias, que aunque en medicina veterinaria no ha sido totalmente establecido presta gran utilidad con relación a la identificación de muchos microorganismos anaerobios relacionados en el área. 
Caracterización de la flora microbiana anaerobia del ciego en la población avícola adulta y sana del Municipio de Los Patios con miras a la producción de probióticos con cepas autóctonas de la región

Adiel Alberto Vasquez Quijano, Jhollman Chacón Rangel, Margarita Caballero Chaparro

Aislamiento: Para evitar contaminación con otro tipo de microorganismos se tomaron al máximo las precauciones pertinentes al trabajo en microbiología. Las muestras se sembraron directamente en caldo tioglicolato y de allí se tomaron alícuotas del fondo del tubo para sembrar y aislar en agar sangre para anaerobios, previa incubación durante 24 horas a $37^{\circ} \mathrm{C}$ en condiciones de anaerobiosis, utilizando el sistema anaeróbico Gaspak.

Al mismo tiempo se tomaron alícuotas del caldo tioglicolato y se sembraron en caldo nutritivo para cultivar la flora aerobia facultativa y evaluar el factor de protección y el factor de infección de la misma.

A las diferentes colonias de anaerobios aisladas se les hizo coloración de Gram y se identificaron con los sistemas CRYSTAL e IDS Rapid ANA II System.

\section{RESULTADOS DE LOS CULTIVOS ANAEROBIOS}

En total se identificaron 24 diferentes bacterias anaerobias de las cuales 15 se han reportado en la literatura sobre probióticos y 9 de ellas son nuevas (Ver tabla 1).

\section{Microorganismos identificados}

Tabla 1. Microorganismos anaerobios identificados por granja, *Microorganismos no reportados en bibliografía

\begin{tabular}{|c|l|l|l|l|l|l|l|l|}
\hline & & G-1 & G-2 & G-3 & G-4 & G-5 & Total \\
\hline 1 & & Actinomycetaceae & $X$ & & & & $X$ & \\
\hline 2 & & Bacillus cereus & & $X$ & & & & \\
\hline 3 & & Bacillus subtilis & & & $X$ & & & \\
\hline 4 & & Bacteroides capillosus & & & & $X$ & & \\
\hline 5 & & Bacteroides vulgatus & $X$ & & $X$ & & & \\
\hline 6 & & Bifidobacterium bifidum & & $X$ & & $X$ & & \\
\hline 7 & & Lactobacillus acidophilus (acid) & $X$ & $X$ & & $X$ & $X$ & \\
\hline 8 & & Lactobacillus bifidu & $X$ & $X$ & $X$ & $X$ & $X$ & \\
\hline 9 & & Lactobacillus casei & & & & & & \\
\hline 10 & & Lactobacillus delbruekii & $X$ & $X$ & & $X$ & $X$ & \\
\hline 11 & & Lactobacillus fermentum & & $X$ & & & & \\
\hline 12 & & Lactobacillus lactis & $X$ & & & $X$ & & \\
\hline 13 & & Saccharomyces cerevisiae & $X$ & $X$ & & & $X$ & \\
\hline 14 & & Streptococcus intermedius & & & $X$ & $X$ & $X$ & \\
\hline 15 & & Streptococcus mutans & & & $\boldsymbol{X}$ & $\boldsymbol{X}$ & & \\
\hline $\mathbf{1 6}$ & $*$ & Mobiluncus curtius & $\boldsymbol{X}$ & $\boldsymbol{X}$ & & & $\boldsymbol{X}$ & \\
\hline $\mathbf{1 7}$ & $*$ & Actinomyces bovis & & & & $\boldsymbol{X}$ & & \\
\hline $\mathbf{1 8}$ & $*$ & Bacteroides thetriomicon & & & & & \\
\hline $\mathbf{1 9}$ & $*$ & Propionibacterium propionicus & $\boldsymbol{X}$ & $\boldsymbol{X}$ & & & & \\
\hline $\mathbf{2 0}$ & $*$ & Actinomyces viscosus & & & & $\boldsymbol{X}$ & $\boldsymbol{X}$ & \\
\hline $\mathbf{2 1}$ & $*$ & Tissierella praeacuta & $\boldsymbol{X}$ & $\boldsymbol{X}$ & $\boldsymbol{X}$ & & & \\
\hline $\mathbf{2 2}$ & $*$ & Bilophila ivadsworthia & & & $\boldsymbol{X}$ & & $\boldsymbol{X}$ & \\
\hline $\mathbf{2 3}$ & $*$ & Capnocytophaga sp. & $\boldsymbol{X}$ & $\boldsymbol{X}$ & $\boldsymbol{X}$ & & & \\
\hline $\mathbf{2 4}$ & $*$ & Propionibacterium guenilosum & $\boldsymbol{X}$ & & $\boldsymbol{X}$ & $\boldsymbol{X}$ & & \\
\hline & & & $\mathbf{1 2}$ & $\mathbf{1 1}$ & $\mathbf{9}$ & $\mathbf{1 1}$ & $\mathbf{1 0}$ & $\mathbf{5 3}$ \\
\hline
\end{tabular}

Vale la pena aclarar que tanto las muestras como las siembras no se refrigeraron puesto que en caso de investigación de anaerobios se ha desechado este proceso por dos razones fundamentales:

a) Es claro que algunas cepas no lo toleran.

b) Los organismos aerobios tienden a incrementarse haciendo inefectivo el procedimiento de anaerobiosis.

Cultivos: La confirmación de la presencia de anaerobios en una muestra está dada por su aislamiento en medios de cultivo. El mayor problema en el aislamiento de anaerobios está dado por la profusión de especies, fácilmente se pueden encontrar docenas de gérmenes anaerobios y de organismos facultativos. Para evitar estos inconvenientes se hizo necesario escoger en forma analítica y sistemática los medios de cultivo adecuados para la obtención de óptimos resultados.

Con estos 24 microorganismos anaerobios identificados y con los cultivos aerobios se produjeron dos probióticos (probiótico de anaerobios y probiótico de aerobios), que se administraron a grupos de 10 pollitos de 4 días de nacidos que se sometieron a una descarga de dosis estandarizada de Salmonella.

\section{EVALUACIÓN DE LA EFECTIVIDAD DEL PROBIOTICO PRODUCIDO}

La evaluación de la eficiencia del tratamiento con el PROBIOTICO, se calcula con base en índices de infección y de protección. (Ver tablas 2, 3 y 4) 
Caracterización de la flora microbiana anaerobia del ciego en la población avícola adulta y sana del Municipio de Los Patios con miras a la producción de probióticos con cepas autóctonas de la región

Adiel Alberto Vasquez Quijano, Jhollman Chacón Rangel, Margarita Caballero Chaparro

Datos obtenidos para grupos de 10 pollitos tratados con probiótico anaerobio y grupos de 10 pollitos control.

Tabla 2. Cantidad de pollitos positivos para salmonella al cabo de cuatro días tras la inoculación.*

Las muestras en que no se detectarón Salmonellas, recibieron un valor de cero (0).

\begin{tabular}{|c|c|c|}
\hline $\begin{array}{c}\text { Dosis Salmonella } \\
\text { (Bacterias } / \mathrm{g} \text { ) }\end{array}$ & $\begin{array}{c}\text { Grupo Control } \\
\text { (No de pollitos positivos) }\end{array}$ & $\begin{array}{c}\text { Grupo Tratado } \\
\text { (No de pollitos positivos) }\end{array}$ \\
\hline $10^{7}$ & 6 & 0 \\
\hline $10^{6}$ & 5 & 1 \\
\hline $10^{5}$ & 3 & 0 \\
\hline $10^{4}$ & 3 & 0 \\
\hline $10^{3}$ & 2 & 0 \\
\hline $10^{2}$ & 1 & 0 \\
\hline $10^{1}$ & 0 & 10 \\
\hline No se aísla $(0)^{*}$ & 0 & \\
\hline
\end{tabular}

Cálculos:

$\mathrm{FI}($ Controles $)=\frac{(6 \times 7)+(5 \times 6)+(3 \times 5)+(3 \times 4)+(2 \times 3)+(1 \times 2)}{10}=\frac{10.7}{10}=10.7$

$\mathrm{FI}($ Tratados $)=(6 \times 1) \div 10=<0.6$

FP

$$
=: \frac{\mathrm{FI}(\text { Controles })}{\mathrm{FI}(\text { Tratados })}=\frac{10.7}{0.6}=>17.83
$$

Valores bajos del Factor de Infección (FI) indican baja proporción de aves infectadas con Salmonella, y bajo nivel de animales portadores; lo contrario ocurre con valores altos de $\mathrm{Fl}$.

En cuanto al Factor de Protección (FP), mientras mayor sea el valor, más eficaz es la protección. Teóricamente, el máximo valor que se obtiene es $>$ 80 , en el supuesto de que todas las aves control tuviesen $10^{8}$ Salmonella / $\mathrm{g}$ en los ciegos, y que todas las aves tratadas fuesen negativas. Es improbable alcanzar este resultado en la práctica: Los valores que se obtienen son mucho menores. Pivnick y col. Citaron valores desde 4.8 hasta 17.5. Aunque es imposible relacionar estos datos directamente con la aplicación comercial del tratamiento, se ha sugerido que un factor de protección $<4$ tendría poca o ninguna posibilidad de reducir las infecciones con Salmonella en las aves domésticas.

Datos obtenidos para grupos de 10 pollitos tratados con mezcla de probiótico anaerobio y aerobio.

Grupos de 10 pollitos control.

Tabla 3. Cantidad de pollitos positivos para Salmonella al cabo de cuatro días tras la inocución.*

Las muestras en que no se detectaron Salmonellas, recibieron un valor de cero (0).

\begin{tabular}{|c|c|c|}
\hline $\begin{array}{c}\text { Dosis Salmonella } \\
\text { (Bacterias } / \mathrm{g})\end{array}$ & $\begin{array}{c}\text { Grupo Control } \\
\text { (No de pollitos positivos) }\end{array}$ & $\begin{array}{c}\text { Grupo Tratado } \\
\text { (No de pollitos positivos) }\end{array}$ \\
\hline $10^{7}$ & 5 & 0 \\
\hline $10^{6}$ & 3 & 0 \\
\hline $10^{5}$ & 3 & 0 \\
\hline $10^{4}$ & 2 & 0 \\
\hline $10^{3}$ & 0 & 0 \\
\hline $10^{2}$ & 1 & 0 \\
\hline $10^{1}$ & 0 & 10 \\
\hline No se aísla $(0)^{*}$ & 0 & \\
\hline
\end{tabular}

Cálculos:

$\mathrm{FI}($ Controles $)=\frac{(5 \times 7)+(3 \times 6)+(3 \times 5)+(2 \times 4)+(1 \times 2)}{10}=\frac{78}{10}=7.8$

$\mathrm{FI}($ Tratados $)=\frac{10 \times 0}{10}<0.1$

$\mathrm{FP} \quad=\frac{\mathrm{FI}(\text { Controles })}{\mathrm{FI}(\text { Tratados })}=\frac{7.8}{0.1}=78.0$

\begin{tabular}{|c|c|c|c|c|c|c|}
\hline & & G-1 & G-2 & G-3 & G-4 & G-5 \\
\hline Probiótico Aerobio & FP & 54 & 45 & 45 & 50 & 49 \\
\hline $\begin{array}{c}\text { Probiótico mixto } \\
\text { (aerobio+anaerobio) }\end{array}$ & FP & 79 & 74 & 67 & 78 & 78 \\
\hline
\end{tabular}

\section{CONCLUSIONES}

- Un buen probiótico se debe preparar con flora aerobia y anaerobia. 
Caracterización de la flora microbiana anaerobia del ciego en la población avícola adulta y sana del Municipio de Los Patios con miras a la producción de probióticos con cepas autóctonas de la región

Adiel Alberto Vasquez Quijano, Jhollman Chacón Rangel, Margarita Caballero Chaparro

- La capacidad protectora del material cecal o fecal varía de ave en ave y de granja en granja.

- Para mejores resultados de protección un probiótico se debe preparar con flora bacteriana autóctona de la región.

- Es importante identificar la flora bacteriana para evitar la propagación de agentes patógenos.

- Los nueve microorganismos nuevos encontrados demuestra que no se han identificado en su totalidad aquellas bacterias con capacidad prebiótica.

\section{BIBLIOGRAFIA}

CAGIGAS, Ada Lydia, y BLANCO, Jorge. Prebióticos y probióticos, una relación beneficiosa. Revista Cubana Alimentación Nutrición 2002;16(1):63-8. Instituto de Nutrición e Higiene de los Alimentos.

AMIGO, Saúl. Evaluación de la actividad probiótica sobre algunos indicadores inmunológicos de un producto de exclusión competitiva en pollos de ceba. Instituto de Investigaciones Avícolas, Ciudad de La Habana, Cuba.

FULLER, R. The importance of lactobacilli in maintaining normal microbial balance in the crop. Br. Poul. Sci. (1977) 18:85-94.

GUILLOT, J.F. The pros and cons of probiotics: Make probiotics work for poultry. Feed Mix Especial 2000.

PÉREZ, HUGO. Una nueva herramienta profiláctica: "La exclusión competitiva en avicultura". VENEZUELA AVICOLA 33, Pág. 11.

JERNIGAN, M.A.; MILES, R.D. \& ARAFA, A.S. (1985) Probiotics in poultry nutrition - a review. World's Poult. Sic. J. 41 : 99 - 107. 52:197-203.

Manual Bayer de Exclusión Competitiva en Aves. http:/ /www.e-campo.com/media/news/nl/default.htm

BRIZUELA, María, BUENO, Lourdes, GUYOT, Nieves, GARCÍA-QUINTANS \& LÓPEZ, Paloma. Evaluación fisiológica y tecnológica de cepas de Lactobacillus con potencialidades prebióticas. Rev. Cubana de Ciencia Avícola, 2001, 25:1- 9

MORIÑIGO, Miguel Ángel. PROBIÓTICOS EN NUTRICIÓN ANIMAL. www.infocarne.com.

MILES, Richard. D. Manipulación de la microflora del tracto gastrointestinal. Departamento de Ciencias Avicolas. Instituto de Ciencias de la Alimentación y Agrícola. 1993.

MOLDER, R.W.A.W. Probiotics and competitive exclusion microflora against Salmonella. Science and Health. Wageningen. The Netherlands. World Poultry Special, May 1996.

MOLDER, R.W.A.W. Probiotics as a tool against Salmonella contamination. Misset Wolrd Poultry 7 (1991), (3) : 36-37.

PAULINA A. SERRANO, MARÍA A. BRIZUELA, ET ALL. Validación del probiótico Problac en aves. Instituto Cubano de Investigaciones de los Derivados de la Caña de Azúcar. Laboratorio de Bromatología y Bioquímica, Instituto de investigaciones Avícolas. email: viiacan@ceniai.inf.cu

PEREZ TORRES, Juan Ramón. Uso de probióticos en aves de jaula. Revista Ornitológica de Pájaros. 1998.

PROBIOTICOS Y AVES. http://www.arrakis.es/ gir/ enfermedad_html.

PORTA, Ramón. Problemática actual de la Salmonella enteritidis. Centre de Sanítat Avĺcola de Catalunya. Mundo Ganadero n 112. Junio de 1999.

SÁNCHEZ GARCÍA, Ada Iris. Empleo de sustancias con actividad probiótica en gallináceas. Universidad de Matanzas, Matanzas, Cuba

Una nueva herramienta profiláctica: "La exclusión competitiva en avicultura" [12-jul-01]. http://www.ecampo.com/media/news/weekly-ag-report/default.cfm

RABSCH, Wolfgang. ET AL. Perspectivas Exclusión competitiva de Salmonella enteritidis por Salmonella gallinarum en pollos. EIE -EID, Vol_6 N_5. 